\title{
Prediction of acoustic foam properties by numerical simulation of polyurethane foaming process
}

\author{
Hichem Abdessalam¹, Boussad Abbès ${ }^{1, a}$, Yuming Li ${ }^{1}$, Ying-Qiao Guo ${ }^{1}$, Elvis Kwassi and Jean-Luc Romain ${ }^{2}$ \\ ${ }^{1}$ Université de Reims Champagne-Ardenne, GRESPI, MPSE, Campus Moulin de la Housse, BP 1039, 51687 Reims Cedex 2, France \\ ${ }^{2}$ Faurecia, Route de Villemontry, 08210 Mouzon, France
}

\begin{abstract}
This work aims to model and to simulate the polyurethane foaming process. Models taking into account the two main chemical reactions of the formation of polyurethane, the exothermic effect of these reactions as well as the thermo-rheo-kinetic coupling characterizing this process are proposed and implemented in the software NOGRIDpoints based on a meshless method (Finite Pointset Method). A prediction of some acoustic foam characteristics is also proposed based on the results of the numerical simulation of the foaming process and semi-phenomenological models.
\end{abstract}

\section{Introduction}

The automotive suppliers produce large amounts of soundproofing parts in flexible polyurethane foam whose shapes are more and more complex in order to satisfy customer demands. The complexity of the parts may cause several types of product defects such as air entrapment, weld lines, unfilled regions in the mould ...etc. affecting the quality of the final part. Thus, the numerical simulation of the foaming process is important for the overall design process to define suitable injection points and air vents to avoid these defects. Different authors [1-5] addressed these issues.

On the other hand, the foamed parts are used in the automotive industry to absorb the maximum amount of sound waves to improve the comfort inside the car. Acoustical performance of the porous materials can be characterized by sound absorption and sound transmission loss. The measurement of these parameters takes lot of time and money. So it is necessary to predict acoustic properties of porous materials by simulation. To predict acoustic performance, five intrinsic parameters i.e., porosity, airflow resistivity, tortuosity, viscous characteristic length and thermal characteristic length are required [6-7]. However, these parameters are highly dependent on the material nature and the foaming process conditions. There is a need to connect the process conditions to the aforementioned parameters to improve the acoustic efficiency of the parts during the part and process design.

This paper is a first tentative to estimate the acoustic properties of the foam using the results of the numerical simulation of foaming process. To do so, a thermo-rheo-kinetic model was developed to simulate the polyurethane foaming process by the Finite Poinset
Method (FPM) and the Johnson-Champoux-Allard semiphenomenological model was used to predict the acoustic foam properties.

\section{Simulation of mould filling with polyurethane foams}

Describing polyurethane (PU) foams as a pseudohomogeneous fluid with continuously changing material properties is a common approach when modelling the mould filling processes [1-4].

The PU foam expansion is governed by the evolution of its density obtained by an empirical equation [8] or by considering the contribution of the chemical reactions, involving the temperature and the viscosity evolution [9$11]$

\subsection{Polyurethane thermo-rheo-kinetic models}

The reactions taking place in PU foams are complex, but can be represented by only two global exothermic reactions: the first gelling reaction, where polyol and isocyanate react to form highly cross-linked polyurethane; and blowing reaction where water and isocyanate react to form urea and carbon dioxide.

Both global reactions are governed by chemical kinetics and are supposed in this work to follow a Piloyan law [12]:

$$
\frac{d r}{d t}=\frac{1}{\tau_{r}} r^{m_{r}}(1-r)^{n_{r}}
$$

where $r$ is the chemical conversion rate of $\mathrm{CO}_{2}$ creation $(r=\alpha)$ or of the gelling reaction $(r=\beta), \tau_{r}$ is the

\footnotetext{
a Corresponding author: boussad.abbes@univ-reims.fr
} 
characteristic time of the reaction, $m_{r}$ and $n_{r}$ are the exponents of the reaction rate.

The foam is considered in this work as a generalized Newtonian fluid with its viscosity depending on the temperature $(T)$, the chemical conversion rate of the gelling reaction $(\beta)$ and the porosity of the foam $(\phi)$ :

$$
\begin{aligned}
\eta(T, \phi, \beta) & =\eta_{0} \exp \left[\frac{E_{\eta}}{R}\left(\frac{1}{T}-\frac{1}{T_{0}}\right)\right] \times \\
& \exp \left(\frac{k(T)}{\phi}\right)\left(\frac{\beta_{g e l}}{\beta_{g e l}-\beta}\right)^{a(T) \beta+b(T)}
\end{aligned}
$$

where $\eta_{0}$ is the viscosity at a reference temperature $\left(T_{0}\right)$, $R$ is ideal gas constant, $E_{\eta}$ is the activation energy, $\beta_{g}$ is the conversion at gel point, $k(T), a(T)$ and $b(T)$ are parameters of the model which generally depend on the temperature.

\subsection{Governing equations}

The general governing equations for compressible Newtonian fluid based on the Stokes' hypothesis include mass conservation, momentum conservation, and energy conservation equations respectively:

$$
\begin{gathered}
\nabla \cdot \mathbf{v}=\phi\left(\frac{1}{\alpha} \frac{d \alpha}{d t}+\frac{1}{T} \frac{d T}{d t}\right) \\
\frac{d(\rho \mathbf{v})}{d t}=-\nabla p+\nabla \cdot \mathbf{s}+\rho \mathbf{g} \\
\frac{d\left(\rho C_{p} T\right)}{d t}=\nabla \cdot(\lambda \nabla T)-(\mathbf{s}: \nabla \mathbf{v})+Q
\end{gathered}
$$

with:

$$
\begin{gathered}
\mathbf{s}=\eta(T, \phi, \beta)\left[(\nabla \mathbf{v})+(\nabla \mathbf{v})^{T}\right] \\
Q=\Delta H_{\beta} \frac{d \beta}{d t}+\Delta H_{\alpha} \frac{d \alpha}{d t}
\end{gathered}
$$

where $\mathbf{v}$ is the velocity vector, $\mathbf{g}$ is the gravity acceleration vector, $\mathbf{s}$ is the deviator part of the stress tensor, $\rho=(1-\phi) \rho_{\mathrm{PU}}+\phi \rho_{\mathrm{CO}_{2}}$ is the density of the foam, $C_{p}=(1-\phi) C_{P U}+\phi C_{C_{2}}$ is the thermal capacity of the foam, $\lambda=(1-\phi) \lambda_{P U}+\phi \lambda_{C_{2}}$ is the thermal conductivity of the foam, $\Delta H_{\alpha}$ is the enthalpy of the reaction of isocyanate with water and $\Delta H_{\beta}$ is the enthalpy of the gelling reaction.

\subsection{Numerical implementation}

Since the 1970s, the meshless formulation was developed from with the emergence of SPH (smoothed particle hydrodynamics) as an alternative to grid-based numerical methods [13]. Later, a set of methods based on this approach have been developed [14]. To avoid the interpolation consistency at the boundary conditions, several solutions have been proposed such as the finite point method (FPM) [15-16]. The fluid domain is discretized by finite number of Lagrangian particles which move with fluid velocity and carry along with them all fluid properties such as density, viscosity, velocity and temperature. The list of neighbour points is determined for each point at each time step through the definition of a zone of influence. Indeed, a smoothing length $h$ is attached to each particle and used to create an interpolation function using a Moving Least Square approximation.

The set of differential equations defined by conservation equations (3)-(5) with appropriate boundary conditions are solved in the NoGrid-points software, where the chemical kinetics, the foam viscosity and the source terms defined by equations (1), (2) and (7) respectively were implemented. A splitting technique is used to solve this highly coupled and non-linear system.

\section{Acoustic properties of PU foam}

The homogeneous foam layer is rather described by the following two intrinsic acoustical properties: the wave number $k(\omega)=\omega(\rho(\omega) / K(\omega))^{1 / 2}$ and the characteristic impedance $Z_{c}(\omega)=(\rho(\omega) K(\omega))^{1 / 2}$. The well-known Johnson-Champoux-Allard semiphenomenological model [6-7] is generally used to predict the frequency behaviour of $\rho$ and $K$ as:

$$
\rho(\omega)=\rho_{0}\left\{\alpha_{\infty}+\frac{\phi \sigma}{j \omega \rho_{0}}\left[1+\frac{j \omega}{v}\left(\frac{2 \alpha_{\infty} \eta}{\Lambda^{2} \phi \sigma}\right)\right]^{1 / 2}\right\}
$$

$$
K(\omega)=P_{0}\left\{1-\frac{\gamma-1}{\gamma}\left(\frac{8 v^{\prime}}{j \omega \Lambda^{\prime 2}}\left[1+\frac{j \omega}{v^{\prime}}\left(\frac{\Lambda^{\prime}}{4}\right)^{2}\right]^{1 / 2}+1\right)^{-1}\right\}^{-1}
$$

where $\omega$ is the angular frequency, $\rho_{0}$ is the fluid density, $\eta$ is the fluid viscosity, $P_{0}$ is the atmospheric pressure, $\gamma$ is the specific heat ratio and $v=\eta / \rho_{0}=P_{\mathrm{r}} v^{\prime}, P_{\mathrm{r}}$ being the Prandtl number.

The macroscopic non-acoustical parameters involved in this model (i.e., static airflow resistivity $\sigma$, tortuosity $\alpha_{\infty}$, viscous $\Lambda$, and thermal $\Lambda^{\prime}$ characteristic lengths) were estimated by the semi-phenomenological model of Dourtes et al. [17] where the foam cell is considered as a tetrakaidecahedron made of 14-sided polyhedron, with six squares faces and eight hexagonal faces (Fig. 1). 


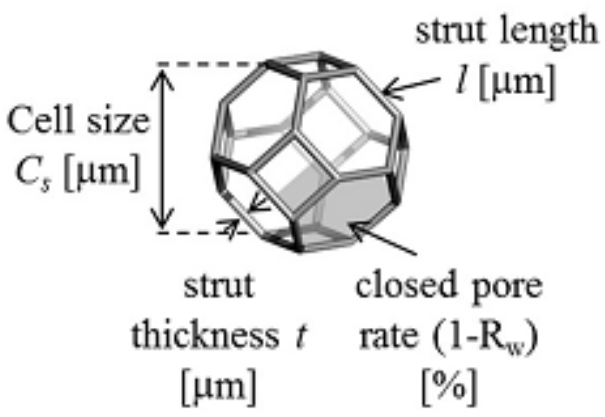

Figure 1. Shape of the idealized unit- [16].

The airflow resistivity is given by:

$$
\sigma=128 \eta\left(C_{r}^{\rho} \frac{A \sqrt{2}}{B}\right)^{2}\left(\frac{1}{C_{s}}\right)^{2}\left(\frac{1}{R_{W}}\right)^{1.1166}
$$

where $C_{s}$ is the cell size, $R_{W}$ is the reticulation rate, $A=C_{s} / \sqrt{2} l$ and $B=l / t$ are two geometric ratios, $\eta$ is the air viscosity, and $C_{s}^{\rho}$ is associated to strut with circular cross-section shape $\left(C_{s}^{\rho}=3 \pi / 8 \sqrt{2}\right)$.

The tortuosity increase with the close pore content $\left(1-R_{W}\right)$ and is given empirically as:

$$
\alpha_{\infty}=1.05\left(\frac{1}{R_{W}}\right)^{0.3802}
$$

The thermal characteristic length $\Lambda^{\prime}$ (also referred to as the hydraulic radius) can be determined from simple geometrical calculations since it is defined as twice the average ratio of the cell volume to their wet surface. It can be written in terms of cell size, reticulation rate and the two geometric ratios ( $A$ and $B$ ) as:

$$
\Lambda^{\prime}=C_{s} \frac{8\left[1-(2 \sqrt{3}-\pi) / B^{2} \sqrt{2}\right] / 3 A}{(1+2 \sqrt{3})-R_{W}(1+2 \sqrt{3}-4 \pi / B \sqrt{3})}
$$

The viscous characteristic length $\Lambda$ is given by the following empirical expression in terms of the closed pore content:

$$
\Lambda=\frac{\Lambda^{\prime}}{1.55\left(\frac{1}{R_{W}}\right)^{0.6763}}
$$

In this work, the foam density $\rho$ and the foam porosity $\phi$ are directly obtained from the simulation of the foaming process.

The Young's modulus of the foam was estimated by the model of Gibson and Ashby [18] where the foam cell is idealized by a cubic shape:

$$
\frac{E^{*}}{E_{s}}=C_{1}\left(\frac{\rho}{\rho_{s}}\right)^{2}
$$

where $E^{*}$ is the Young's modulus of the foam, $E_{s}$ is the Young's modulus of the solid polyurethane, $\rho$ is the foam density, $\rho_{s}$ is the density of the solid polyurethane, $C_{1}$ is the model parameter.

The normal-incidence sound absorption coefficient of the foam is given by:

$$
\alpha=1-\left|\frac{Z_{s}-\rho_{0} c_{0}}{Z_{s}+\rho_{0} c_{0}}\right|^{2}
$$

where $c_{0}$ is the speed of sound in the air and $Z_{\mathrm{s}}$ is the normal-incidence surface impedance. For a layer of fluid of thickness $\mathrm{h}$ and backed by an impervious rigid wall, this impedance is given by [19]:

$$
Z_{S}=-j Z_{c} \cot (k h) / \phi
$$

\section{Results and discussion}

\subsection{Mould filling results}

The square panel mould shown in Fig. 2 is used to validate the implemented models. By fixing the mass flow to $200 \mathrm{~g} / \mathrm{s}$, short shot foams are obtained by using different injection times and by varying the mould cavity thickness from $8 \mathrm{~mm}$ to $20 \mathrm{~mm}$.

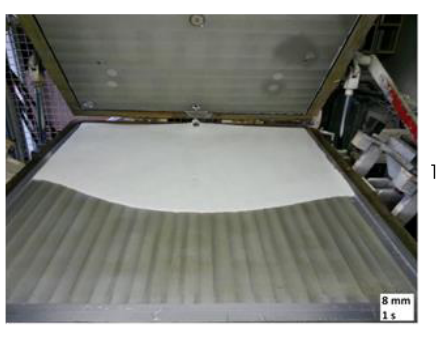

Figure 2. Square panel mould.

Thanks to the symmetry of the problem only a half of the mould has been modelled. Initially, the fluid domain is free from particles and a cylindrical injector is filled with a set of particles defining the free surface at the injection point. A constant volumetric flow rate is imposed at the injector inlet. A set of fixed particles are automatically affected to the walls in NoGrid-points. When the mould is completely filled the number of particles reaches 305000 .

An inverse identification procedure for the model parameters was developed in a previous work [18] and herein used for the simulation of the PU foaming process.

The experimental and numerical results obtained for the mould cavity thickness of $12 \mathrm{~mm}$ and injections times of $1 \mathrm{~s}$ and $2 \mathrm{~s}$ are shown in Fig. 3. One can notice that 
FPM simulations capture accurately the front position showing a good agreement with the corresponding experimental results.
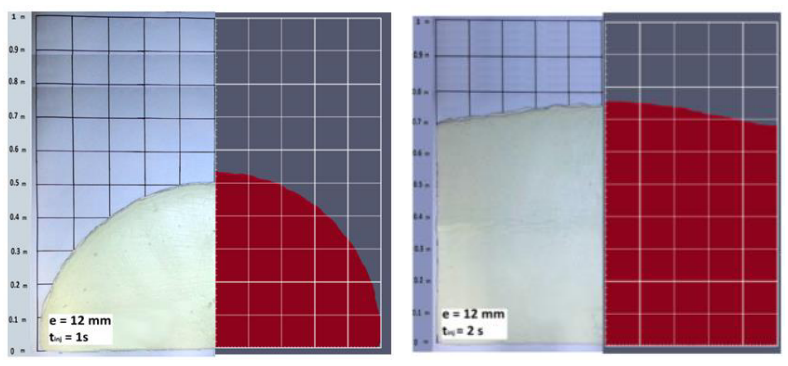

Figure 3. Comparison between experimental and numerical results for short shot foams of the mould cavity.

Fig. 4 shows the temperature, viscosity and density profiles of through the thickness of the foam. The temperature increases from the fixed mould temperature $\left(60^{\circ} \mathrm{C}\right)$ to its maximum value at the middle of the thickness because of the exothermic effects of the two global reactions. Since the viscosity depends on the temperature, there is also a viscosity gradient in the thickness. The profile of the foam density is inverted with respect to that of the temperature, since the density is inversely proportional to the porosity which is proportional to the temperature. It is then found that the density is minimum at middle of the thickness. This result was confirmed by using an optical microscope observation of the foam structure across the section of the short shot (Fig. 5).
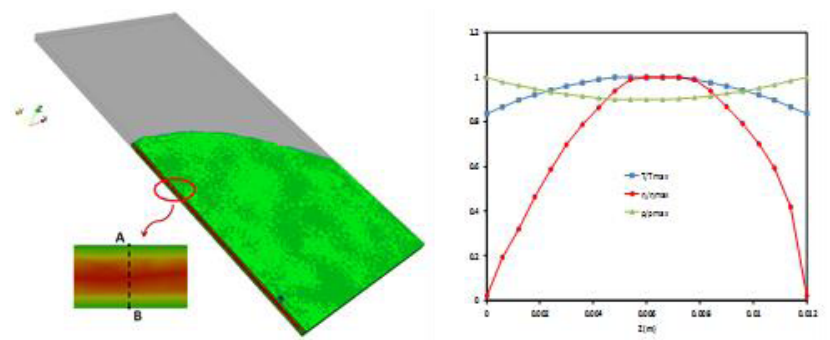

Figure 4. Temperature, viscosity and density profiles through foam thickness.

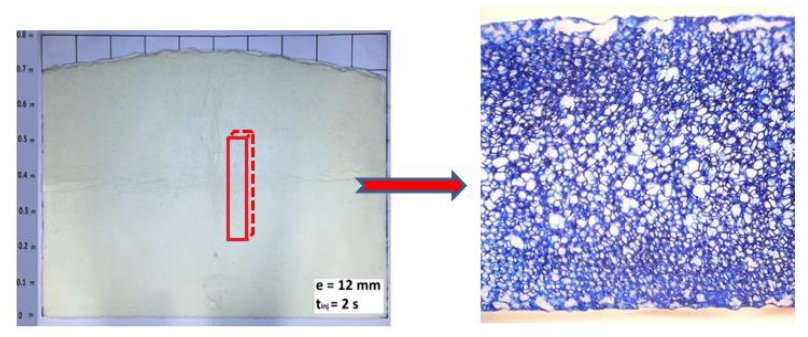

Figure 5. Optical microscope observation of the foam structure across section of the short shot.

\subsection{Acoustic results}

The model parameters values of the acoustic semiphenomenological model are listed in Table 1. Fig. 6 shows the iso-values of the porosity and the Young's modulus obtained at the end of the short shot simulation showing in particular the presence of a gradient across the thickness.

Table 1. Semi-phenomenological model parameters.

\begin{tabular}{|c|c|}
\hline Parameter & Value \\
\hline$E_{\mathrm{s}}$ & $0.03 \mathrm{GPa}$ \\
\hline$\rho_{\mathrm{s}}$ & $1200 \mathrm{~kg} / \mathrm{m}^{3}$ \\
\hline$\eta$ & $1.8110^{-5} \mathrm{~Pa} . \mathrm{s}$ \\
\hline$C_{\mathrm{s}}$ & $900 \mu \mathrm{m}$ \\
\hline$C_{1}$ & 0.63 \\
\hline$R_{\mathrm{W}}$ & 1 \\
\hline$A$ & 2.33 \\
\hline$B$ & 2.13 \\
\hline
\end{tabular}
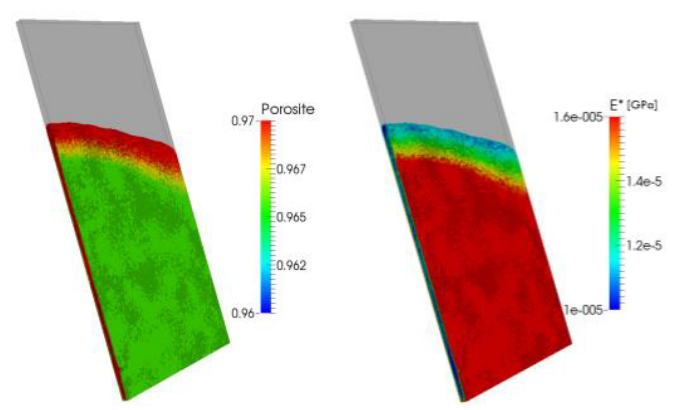

Figure 6. Iso-values of porosity and Young's modulus.
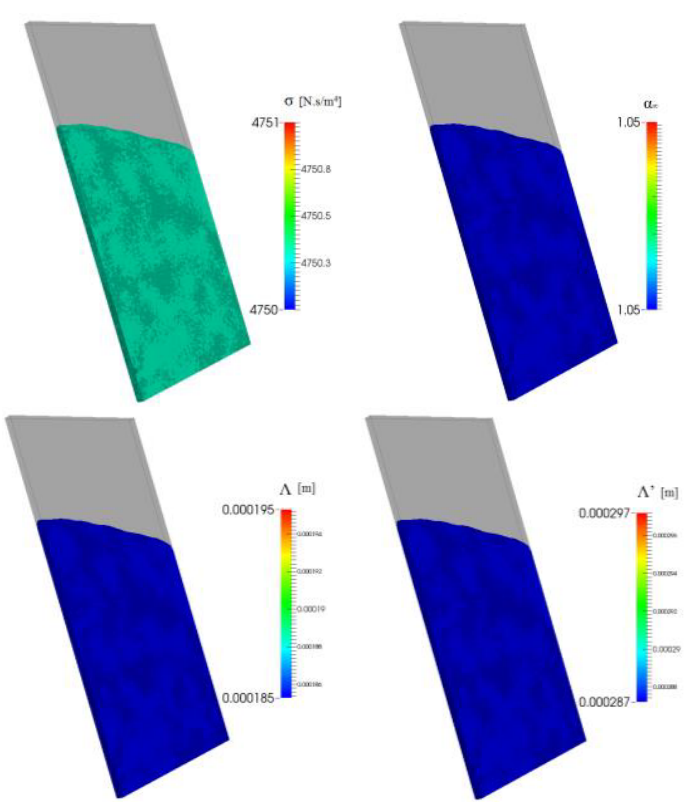

Figure 7. Iso-values of static airflow resistivity, tortuosity, viscous and thermal characteristic lengths. 
Constant iso-values of the macroscopic non-acoustical parameters (i.e., static airflow resistivity $\sigma$, tortuosity $\alpha_{\infty}$, viscous $\Lambda$, and thermal $\Lambda^{\prime}$ characteristic lengths) are shown in Fig. 7.

Fig. 8 presents the simulation of the sound absorption coefficient of the $12 \mathrm{~mm}$ thick foam derived from the Johnson-Champoux-Allard model using the computed non-acoustic properties.

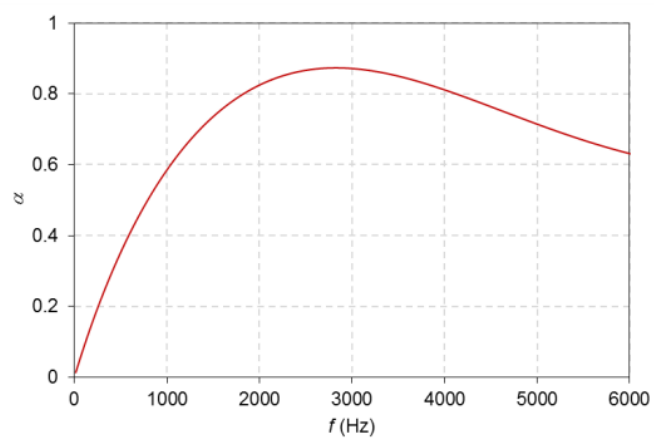

Figure 8. Sound absorption coefficient of PU foam.

\section{Conclusions}

The foaming process of polyurethane was physically modelled by considering the expansion of a compressible quasi-homogeneous continuous mixture. The model was implemented in the FPM framework. The comparison between the simulation and the experimental results was achieved through the foaming process of set of short shots using a panel mould cavity. The validation consisted in comparing the flow front positions obtained experimentally with the numerical ones. The results show a good agreement between the experimental and the numerical results. A prediction of some acoustic foam characteristics based on the results of the numerical simulation of the foaming process by using a semi-phenomenological model were also proposed. The proposed models and methodology enable to largely reduce the development delay and costs by quickly determining the foaming process parameters and by using less mould prototypes.

\section{References}

1. Y.B. Kim, K.D. Kim, S.E. Hong, J.G. Kim, M.H. Park, J.H. Kim, J.K. Kweon, J. Cell. Plast., 41, 251 (2005).

2. S. Seo, J.A. Youn, Polymer, 46, 6482 (2005).

3. J. Bikard, J. Bruchon, T. Coupez, L. Silva, Colloids Surf. A, 49, 309 (2007).

4. S. Geier, C. Winkler, M. Piesche, Chem. Eng. Technol., 32, 1438 (2009).

5. H. Abdessalam, B. Abbès, Y.M. Li, Y.Q. Guo, E. Kwassi, J.L. Romain, Int. J. Mater. Form., 9(1), 85 (2016).

6. D.L. Johnson, J. Koplik, R. Dashen, J. Fluid Mech., 176, 379 (1987).
7. Y. Champoux, J.-F. Allard, J. Appl. Phys., 70(4), 1975 (1991).

8. W.H. Lee, S.W. Lee, T.J. Kang, K. Chung, J.R. Youn, Fibers Polym., 3, 159 (2002).

9. (9)S.A. Baser, D.V. Khakhar, Polym. Eng. Sci., 34, 642 (1994).

10. J. Bikard, J. Bruchon, T. Coupez, B. Vergnes, J. Mater. Sci., 40, 5875 (2005).c

11. R. Bouayad, J. Bikard, J. F. Agassant, Int. J. Mater. Form., 2, 243 (2009).

12. G.O. Piloyan, I.D. Ryabchikov, O.S. Novikora, Nature, 212, 1229 (1966).

13. L.B. Lucy, Astron. J., 83, 1013 (1977).

14. S. Li and W.K. Liu, Meshfree Particle Methods (Springer, Berlin, 2007).

15. E. Oñate, S. Idelsohn, O.C. Zienkiewicz OC, L.R. Taylor and C. Sacco, Comput. Meth. Appl. Mech. Eng., 139, 315 (1996).

16. J. Kuhnert, General smoothed particle hydrodynamics, $\mathrm{PhD}$ Thesis, (University of Kaiserslautern, Germany, 1999).

17. O. Doutres, N. Atalla, K. Dong, J. Appl. Phys., 113, 054901 (2013).

18. L.J. Gibson, M.F. Ashby, Cellular solids: Structure and Properties, (Cambridge University Press, Cambridge, 1988).

19. J.F. Allard, N. Atalla, Propagation of Sound in Porous Media: Modeling Sound Absorbing Materials, 2nd ed. (Willey, New York, 2009). 